\title{
Continuous Bilateral Posterior Lumbar Plexus Block with a Disposable Infusion Pump. Case Report
}

\author{
Luiz Eduardo Imbelloni, TSA ${ }^{1}$, Eneida Maria Vieira ${ }^{2}$, Fábio Stuchhi Devito ${ }^{3}$, Eliana Marisa Ganem, TSA 4
}

\begin{abstract}
Summary: Imbelloni LE, Vieira EM, Devito FS, Ganem EM - Continuous Bilateral Posterior Lumbar Plexus Block with a Disposable Infusion Pump. Case Report.

Background and objectives: The number of bilateral total hip arthroplasties (THA) has been increasing every year. Postoperative analgesia by continuous perineural infusion of local anesthetic has been shown favorable results when compared to systemic analgesia. The use of elastomeric pumps has increased patient satisfaction when compared to electronic models. The objective of this report was to describe a case of continuous bilateral posterior lumbar plexus block with an elastomeric infusion pump in a patient submitted to bilateral hip arthroplasty.

Case report: This is a 46 year-old female patient weighing $65 \mathrm{~kg}, 162 \mathrm{~cm}$, with rheumatoid arthritis and hypertension, physical status ASA II, scheduled for bilateral THA in a single stage. She had been on corticosteroids for 13 years. Hemoglobin $=10.1$ g. $\mathrm{dL}^{-1}$, hematocrit $=32.7 \%$. Routine monitoring. Spinal anesthesia with $15 \mathrm{mg}$ of $0.5 \%$ isobaric bupivacaine. General anesthesia with propofol (PFS) and remifentanil, and intubation without neuromuscular blockers. Right THA and, at the end, lumbar plexus block with a stimulator and a set of $150 \mathrm{~mm}$ needle and injection of 20 $\mathrm{mL}$ of $0.2 \%$ bupivacaine and introduction of a catheter. Left THA and, at the end, the same procedure. Anesthetic dispersion and contrast were investigated. Elastomeric pump was installed with $0.1 \%$ bupivacaine $(400 \mathrm{~mL})$ at a rate of $14 \mathrm{~mL}^{-1} \mathrm{~h}^{-1}$. The patient was transferred to the Intensive Care Unit (ICU). After 24 hour, a new pump was installed with the same solution. She did not receive any boluses for 50 hours. After removal of the catheter, pain was controlled with oral ketoprofen and dypirone.
\end{abstract}

Conclusions: Continuous peripheral blockade with infusion of $0.1 \%$ bupivacaine with elastomeric pumps is a safe and effective procedure in adults.

Keywords: Infusion Pumps; Anesthesia, Conduction; Lumbosacral plexus; Bupivacaine; Arthroplasty, Replacement, Hip.

[Rev Bras Anestesiol 2011;61(2): 211-217] @Elsevier Editora Ltda.

\section{INTRODUCTION}

Pain that requires total hip arthroplasty (THA) has many etiologies, and many of them with bilateral incidence. Large orthopedic surgeries can be associated with severe pain, which is difficult to treat requiring prolonged hospitalization. Continuous peripheral nerve block (CPNB) can provide prolonged postoperative analgesia, avoiding many of the collateral effects of opioid analgesia. A continuous infusion of local anesthetic through an epidural catheter can provide longer lasting analgesia than single injection. Elastomeric pumps have an inflatable elastomer, release tube, and a bacterial filter. The combination of epidural catheter and portable infusion pump can be used in outpatients for pain control, therefore avoiding an unnecessary hospitalization ${ }^{1}$. The objective of the present

Received from Instituto de Anestesia Regional São José do Rio Preto, SP, Brazil.

1. Anesthesiologist;

2. Professor of Fundação Faculdade de Medicina de São José do Rio Preto

3. Professor; Physician of the Serviço de Ortopedia do Hospital de Base de São José do

Rio Preto

4. Assisting Professor of the Departamento de Anestesiologia, Faculdade de Medicina de

Botucatu, Universidade Estadual de São Paulo

Submitted on March 17,2010.

Approved on November 10, 2010.

Correspondence to:

Dr. Luiz Eduardo Imbelloni

Av. Epitácio Pessoa, 2.356/203

Lagoa

22411-072 - Rio de Janeiro, Brazil

E-mail:dr.imbelloni@terra.com.br report was to describe a patient submitted to posterior lumbar CPNB with a portable elastomeric device in a patient undergoing bilateral THA.

\section{CASE REPORT}

A 46-year old female with rheumatoid arthritis $(65 \mathrm{~kg}, 162 \mathrm{~cm})$, physical status ASA II, scheduled for bilateral THA in a single stage. She had been on corticosteroids for 13 years. Her medical history included hypertension, which was treated with enalapril (angiotensin-converting enzyme inhibitor). Her blood test showed moderate anemia (hemoglobin $=10.1 \mathrm{~g} \cdot \mathrm{dL}^{-1}$, hematocrit $=32.7 \%$ ).

The patient was evaluated and signed the informed consent to undergo THA under posterior lumbar plexus CPNB. Prolonged analgesia and potential risks of using both catheters were discussed with the patient and family members. Due to the duration of the surgery (estimated 4 hours) and the lateral decubitus required for the surgery, simple spinal anesthesia associated with general anesthesia was performed.

After placement of routine monitoring, spinal anesthesia was performed in the $L_{3}-L_{4}$ space through the median approach with the patient in the sitting position with a $27 \mathrm{G}$ Quincke needle; $15 \mathrm{mg}$ of isobaric $0.5 \%$ bupivacaine were injected. After the blockade, general anesthesia was performed with propofol (PFS) and remifentanil. A 7.5 (ID) endotracheal tube was used for tracheal intubation without neuromuscular blocker. 
Initially, the patient was placed in left lateral decubitus for right arthroplasty. At the end of the procedure, access to the lumbar plexus was achieved using a stimulator (HNS12 BBraun, Melsungen) connected to a 150-mm 18G Tuohy type needle (B.Braun Melsungen). The needle was advanced and the quadriceps was stimulated with a $0.5 \mathrm{~mA}$ current until a response was observed. After negative aspiration of blood, $20 \mathrm{~mL}$ of $0.2 \%$ bupivacaine were injected through the extension connector of the needle. Maintaining the needle in the same position, a 20G catheter was introduced $7 \mathrm{~cm}$ in the psoas compartment, being fixed in place with adhesive material and an occlusive dressing.

The patient was placed in the right lateral decubitus and left THA was initiated. Similarly, $20 \mathrm{~mL}$ of $0.2 \%$ bupivacaine were injected and a similar catheter was introduced.

At the end of the procedure, the patient was placed in the supine position. She recovered from the general anesthesia and was extubated. Approximately $20 \mathrm{~mL}$ of contrast (iohexol with $300 \mathrm{mg} \cdot \mathrm{mL}^{-1}$ - Ominipaque ${ }^{\circledR}$ ) were injected in both catheters to study the dispersion of the local anesthetic, and an anteroposterior X-ray of the psoas compartment was performed within one minute. Both catheters were in the psoas compartment (Figure 1). Before her discharge, a disposable elastomeric pump (Easypump ${ }^{\circledR}$, B.Braun, Germany) containing $400 \mathrm{~mL}$ of $0.1 \%$ bupivacaine was connected to the catheters of both psoas compartments (Figure 2). The pump was programmed for infusion at a rate of $14 \mathrm{~mL} . \mathrm{h}^{-1}$. At the end, the patient was transferred to the Intensive Care Unit (ICU). She received instructions to trigger the bolus device, which is part of the pump, in case of severe pain. Toxicity symptoms of the local anesthetic were recorded. Twenty-four hours later, a new elastomeric pump with the same solution (volume, concentration, and rate of infusion) was installed, which remained for another 25 hours. During the 50 postoperative hours, boluses were not necessary. Both catheters were removed without intercurrences. After catheter removal, pain was controlled with oral ketoprofen and dypirone.

During the procedure, $1,500 \mathrm{~mL}$ of crystalloids, $500 \mathrm{~mL}$ of Voluven $^{\circledR}$ (6\% hydroxyethyl starch $130 / 0.4$ in $0.9 \%$ saline), and tow units of packed-red blood cells were administered. The urinary catheter drained $600 \mathrm{~mL}$ of urine during the procedure.

\section{DISCUSSION}

The use of bilateral lumbar catheters for bilateral THA has not been reported. Since it administers the local anesthetic close to the lumbar plexus, analgesia can be prolonged especially in patients undergoing THA. The results of this case demonstrate that elastomeric pump for CPNB with infusion of local anesthetic in adult patients is an effective and safe analgesia method after some large orthopedic procedures. The use of rescue analgesia was minimal.

The incidence of bilateral THA is a procedure that has been increasing, reducing hospitalization. During THA the patient can remain in lateral decubitus or supine depending on the

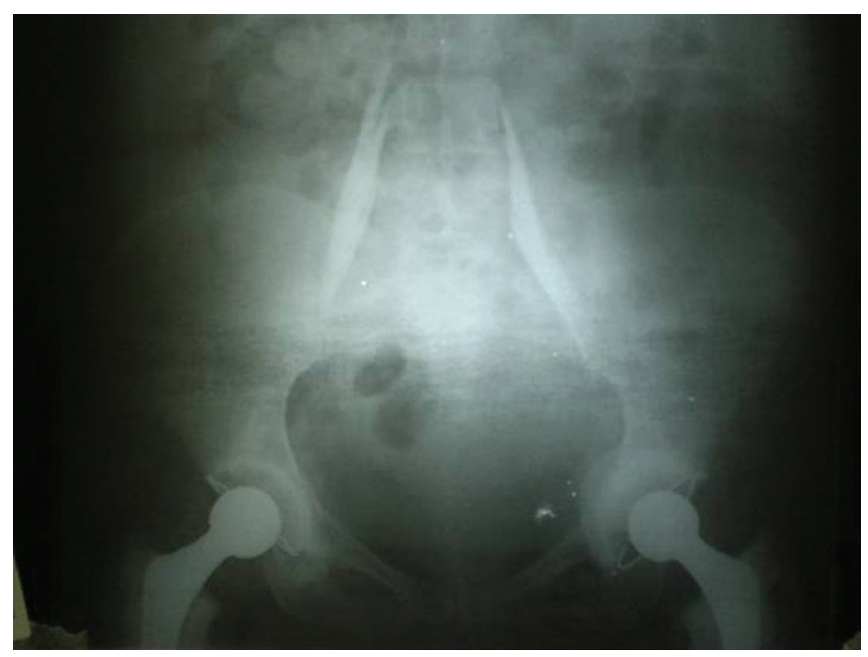

Figure 1 - Catheters Inside the Psoas Compartment.

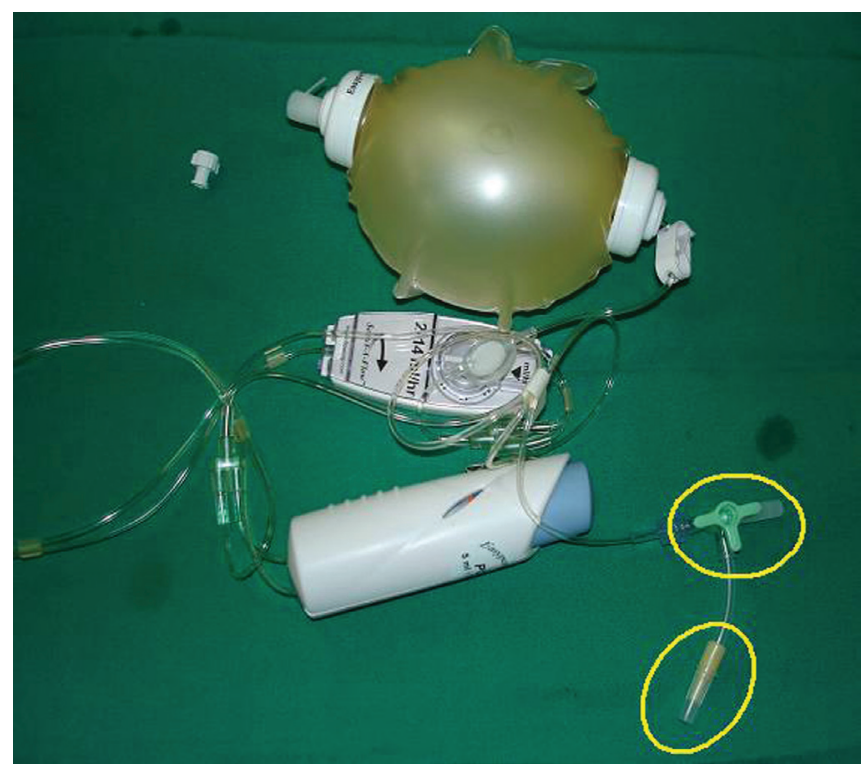

Figure 2 - Disposable Elastomeric Pump (Easypump ${ }^{\circledR}$, B.Braun, Germany).

preference of the surgeon (in this case, the patient was in lateral decubitus).

Psoas compartment block was the technique used for continuous analgesia. This blockade is performed due to the location of the psoas muscle at the level of the $4^{\text {th }}$ and $5^{\text {th }}$ lumbar vertebrae. The local anesthetic is deposited, blocking all lumbar plexus nerves and some roots of the sacral plexus. This approach is superior to the paravascular inguinal technique ${ }^{2}$, especially due to the greater success in blocking the ilioinguinal, genitofemoral, lateral cutaneous femoral, and obturator nerves. For continuous analgesia, the posterior approach offers less chances of catheter dislocation when compared to the anterior approach, since the paravertebral muscle allows better fixation of the catheter ${ }^{3}$. In a recent prospective 
study evaluating the efficacy of continuous analgesia of the psoas compartment $\left(0.2 \%\right.$ ropivacaína at $\left.0.15 \mathrm{~mL} \cdot \mathrm{kg}^{-1} \cdot \mathrm{h}^{-1}\right)$ after THA, a success rate of $97 \%$ in catheter placement and $94 \%$ of excellent postoperative analgesia, without the need of supplementation with systemic opioid were reported ${ }^{4}$. Lidocaine, bupivacaine, and ropivacaína have been used as local anesthetics for continuous plexus analgesia, but bupivacaine and ropivacaína are used more often. The use of bupivacaine $(0.1 \%$ to $0.25 \%)$ did not result in toxic blood levels in postoperative analgesia for $24-72$ hours ${ }^{5}$, the same dose of the present case. The venous level of bupivacaine during continuous lumbar plexus analgesia was $0.5-1.8 \mu \mathrm{g} \cdot \mathrm{mL}^{-16}$, while levels above $2 \mu \mathrm{g} \cdot \mathrm{mL}^{-2}$ are considered toxic.

Bilateral psoas compartment block was performed with a peripheral nerve stimulator and we also observed the distribution of contrast inside those muscles. The distribution of contrast showed a higher level on the right side and lower on the left side, and it seemed to have invaded the lumbosacral territory.

The use of elastomeric pump in bilateral CPNB for postoperative analgesia has several advantages. Portable pumps are used for infusion of anesthetics during continuous regional analgesia and they are increasingly more accepted. Those pumps are frequently used in outpatients without medical supervision. If the elastomeric pump is connected to a catheter, the release of the agent may vary according to its extension. This report demonstrated that the patient received adequate analgesia in both catheters. The rate of the flow of anesthetics is dictated by the elastomeric reservoir and the gauge of the tube, which prevents the infusion of a large bolus and over- dose. Although the pump has its own bacterial filter, a second filter was used to increase patient safety. The elastomeric pump has several advantages over the electronic pump, including: portability, ease of use, and few technical problems, such as undesirable alarms ${ }^{7}$.

Continuous epidural analgesia can be a technical alternative for the treatment of pain after large orthopedic surgeries. Unlike the epidural block, psoas compartment block is considered limitative in cases of sympathectomy and, posteriorly, for eventual hemodynamic changes ${ }^{8}$. Peripheral nerve blocks cause less nausea/vomiting and urinary retention than neuraxial blocks ${ }^{9}$. Contraindications include infection at the site of the blockade and allergy to analgesics or anesthetics. The use of peripheral nerve blocks has been considered in patients on anticoagulants. However, its use in procedures, such as psoas compartment block, should be discussed due to the possibility of retroperitoneal hematoma.

Peripheral nerve block is a simple, safe, and effective technique for perioperative anesthesia and with the use of CPNB and a pump it provides excellent postoperative pain control. Continuous peripheral nerve block has been routinely indicated in some services; however, bilateral continuous psoas compartment block had not been reported yet. The extension of use of local anesthetics to control of postoperative pain has been reported in several countries, with several advantages over commonly used methods, including ease to manage, safe, low cost, and effective. We conclude that continuous bilateral peripheral nerve block with infusion of $0.1 \%$ bupivacaine with elastomeric pump is a safe and effective procedure in adults. 